\title{
INVESTIGACIÓN/RESEARCH
}

Recibido: 20/11/2015 Aceptado: 10/02/2016 Publicado: 15/03/2016

\section{¿CÓMO ANALIZAR LAS CARACTERÍSTICAS DE LOS DEPARTAMENTOS DE COMUNICACIÓN EN LOS HOSPITALES Y SUS CONSECUENCIAS EN EL CIUDADANO? EJEMPLO METODOLÓGICO}

Lorena Busto Salinas ${ }^{1}$ : Universidad de Burgos. España Ibusto@ubu.es

RESUMEN: El artículo expone la metodología utilizada para averiguar la calidad de las relaciones públicas en los hospitales, así como para determinar si el número de departamentos de comunicación influye en las características de esta práctica y en las informaciones aparecidas en prensa y si existen diferencias entre el ámbito público y el privado y entre un gabinete interno y una empresa externa. Además, se ha estudiado si una mayor actividad comunicativa mantiene una correlación con la participación de los ciudadanos en las noticias y en la valoración y satisfacción hacia el sistema sanitario. Para ello, se han tomado como objeto de estudio dos comunidades de España (Castilla y León y Galicia) con similares características sociales, económicas y sanitarias, pero con una estructura comunicativa diferente. Se han realizado entrevistas personales y una encuesta a los encargados de comunicación de cada hospital. Asimismo, se ha realizado un análisis de prensa, un estudio de la participación de los ciudadanos en las noticias en internet y un análisis de la valoración y satisfacción hacia la sanidad. No se han encontrado evidencias de que la competencia comunicativa provoque unas mejores relaciones públicas, pero se ha detectado que la calidad de las noticias es superior en los lugares que cuentan con un mayor número de gabinetes de comunicación y que los ciudadanos de estas zonas votan y comparten más a menudo las informaciones. Asimismo, consideran la salud y la sanidad temas más importantes, tienen una mayor valoración de la sanidad pública y elegirían antes esta titularidad.

PALABRAS CLAVE: relaciones públicas, comunicación sanitaria, análisis de prensa, análisis cuantitativo, análisis cualitativo, comportamiento del consumidor, salud.

\footnotetext{
${ }^{1}$ Lorena Busto Salinas. Doctora en Comunicación, es profesora de comunicación internacional en la Universidad Pontificia de Comillas. Sus líneas de investigación giran en torno a las relaciones públicas, la comunicación sanitaria, la divulgación del patrimonio, la comunicación institucional y el análisis de prensa.

Correo: Ibusto@ubu.es
} 


\title{
HOW TO ANALYZE COMMUNICATION DEPARTMENTS IN HOSPITALS AND THEIR CONSEQUENCES ON THE CITIZENS? EXAMPLE OF METHODOLOGY
}

\begin{abstract}
The article exposes the methodology used to study the quality of public relations in hospitals, as well as to determine if the number of communication departments has an influence on its quality and on new stories, and if there are any differences between the public and private sector and between internal departments and external companies. In addition, it has been studied if hospital communication influences citizens to participate in news stories and if it affects their assessment and satisfaction with the healthcare system. Two communities of Spain (Castilla y Leon and Galicia) with similar social, economic and healthcare system characteristics, but with a different communicative structure, have been chosen as objects of study. Personal interviews and a survey to communication officers of each hospital have been conducted, as well as a press analysis. In addition, an official health report has been analyzed to determine the satisfaction, assessment and selection of health centers. No evidence has been found supporting the idea that the communicative competence produces better public relations. However, it has been noted that the quality of news stories is higher in places with a greater number of press offices. In addition, citizens of these regions vote and share information more often, they consider health issues most important, they have a greater assessment of the public healthcare system and would choose this ownership over private centers.
\end{abstract}

KEYWORDS: public relations, health communication, press analysis, quantitative analysis, qualitative analyses, consumer behavior, health.

\section{INTRODUCCIÓN}

Los asuntos sobre salud y sanidad son cada vez más trascendentes para los ciudadanos, tal y como muestran diversos estudios de opinión (Cangelosi; Ranelli; Markham, 2009; Castiel; Álvarez-Dardet, 2005; Centro de Investigaciones Sociológicas, 2012; Len-Ríos; Hinnant; Park, 2009; Pew Research Center, 2004; Weaver-Lariscy; Avery; Sohn, 2010). Los pacientes ahora se informan más acerca de sus necesidades y ya no se someten únicamente a las instrucciones de su médico, puesto que adoptan un papel más activo en las decisiones relacionadas con su salud (Del-Pozo-Irribarría; Ferreras-Oleffe, 2011; Freberg; Palenchar; Veil, 2013; Gbadeyan, 2010; Thomas, 2005; Wrenn, 2002). El aumento de documentos sobre esta temática, así como de fuentes de información, ha podido contribuir (Amador-Romero, 2004; Avery et al, 2010; Brodie; Glynn; Van Durme, 2002; Eggener, 1998; Observatorio de la Comunicación Científica, 2008; Roessler, 2007; Viswanath, 2006). Y es que las noticias aparecidas en los medios de comunicación se han multiplicado en los últimos años, aunque estos no son la única fuente para informarse sobre asuntos sanitarios. En la actualidad, las personas aprenden sobre medicina y salud a través de familiares y amigos, publicidad, series de televisión, páginas web, foros, blogs, redes sociales, folletos, etc (Amador-Romero, 2004; Avery et al., 2010; Freberg et al., 2013; Jiménez- 
Pernett et al, 2009; Kreps; Bonaguro; Query, 1998; Lupiáñez-Villanueva, 2009; Roessler, 2007; Wrenn, 2002).

En consecuencia, la comunicación en el ámbito sanitario es cada vez más necesaria (Anand \& Chakravarti, 1981; Costa Sánchez, 2012; Díaz, 2011; Henderson, 2005; Hibbard, Stockard, \& Tusler, 2005; Keller et al., 2014; Kirdar, 2007; Meath, 2006; Menéndez Prieto \& Vadillo Olmo, 2010; Pham, Coughlan, \& O'Malley, 2006; Scott, Vojir, Jones, \& Moore, 2005; Springston \& Weaver Lariscy, 2005). El público de los centros dedicados a la salud ya no es simplemente el paciente, sino que otros grupos, como los empleados, los familiares y visitas, la comunidad, las agrupaciones, etc., también son relevantes (Anand; Chakravarti, 1981; Balima, 2006; Díaz, 2011; Gordon; Kelly, 1999; Gudykunst; Kim, 1992; Guy et al, 2007; Igartua-Perosanz, 2006; Ivanov; Sims; Parker, 2013; Kirdar, 2007; Kreps; Kunimoto, 1994; Kreps, 2008; Kurtz, 1969; Springston; Champion, 2004; Weaver-Lariscy; Springston, 2007; Youmans; Schillinger, 2003). Esto cobra especial importancia en el caso de los centros de atención especializada, dada su gran capacidad humana y tecnológica, desde donde puede proporcionarse abundante información tanto de divulgación sanitaria como de actividad hospitalaria. Los departamentos de comunicación de estos centros constituyen un nexo entre la organización y sus diferentes públicos. Entre sus labores se encuentra conocer y cubrir las necesidades de los ciudadanos, transmitir información fiable, servir de enlace entre los periodistas y la comunidad científica, completar o corregir informaciones erróneas, etc.

\section{OBJETIVOS}

No obstante, hasta el momento, no existen estudios que reflejen específicamente las características de los departamentos de comunicación que existen en los centros sanitarios ni tampoco los beneficios que conllevan, tanto por la información que divulgan los medios de comunicación como por las consecuencias para la población. Precisamente, esos son los principales objetivos de la tesis doctoral titulada "La comunicación sanitaria y sus implicaciones en las actitudes e información accesible a los ciudadanos. Estudio comparativo entre Castilla y León y Galicia", defendida por la autora de este artículo en la Universidad de Burgos en 2015, y cuya metodología se expone en este artículo.

Antes de establecer un método de trabajo, se ha diseñado una serie de hipótesis. Estas suposiciones tratan sobre la presencia y características de los departamentos de comunicación en los hospitales y en las consecuencias que estos tienen tanto en la información publicada en prensa como en las percepciones de los ciudadanos acerca de los temas médicos. Tanto a nivel general como enfrentando las dos comunidades analizadas, este estudio se estructura según las siguientes hipótesis:

H1. Debido a la competencia comunicativa, cuando una gran parte de los hospitales de una región posee departamentos de comunicación, las relaciones públicas que se adoptan deberían ser más excelentes. 
H2. La presencia de departamentos de comunicación en los hospitales debe tener una repercusión en el número y calidad de las informaciones sobre salud y sanidad que publican los medios de comunicación de la región.

H3. Los ciudadanos de las zonas que cuentan con departamentos de comunicación en la mayor parte de los hospitales han de ser más activos en el consumo de información sanitaria y salud, en el sentido de que comentan más frecuentemente estos temas, los valoran y los comparten con sus conocidos.

H4. Los ciudadanos de las zonas en las que existen departamentos de comunicación deberían tener una mejor valoración y un grado de satisfacción más alto hacia el sistema sanitario.

H5. Los departamentos de comunicación de los hospitales públicos y de los privados pueden tener diferencias en cuanto a su interés por la opinión pública, en el uso de métodos más o menos tradicionales y en las nuevas acciones de comunicación.

H6. Los hospitales gestionados por empresas externas de comunicación ejercerían unas relaciones públicas más excelentes que aquellos que cuentan con uno o dos empleados en plantilla debido a la diversidad de personal.

\section{METODOLOGÍA}

Para dar respuesta a las hipótesis formuladas, se han seleccionado dos comunidades autónomas de España con similares características sociales, económicas y sanitarias pero con una estructura de comunicación diferente. Concretamente, se han comparado Castilla y León y Galicia. Por ejemplo, en la comunidad castellana habitan 2.765.940 personas y en la gallega, 2.519.875 (Instituto Nacional de Estadística (INE), 2013). La proporción de mujeres y hombres, así como la edad de la población también es muy similar (Instituto Nacional de Estadística (INE), 2013). El producto interior bruto de la región castellana en 2012 es de 21.994 euros por habitante y en la gallega, de 20.330 (Instituto Nacional de Estadística (INE), 2012). En cuanto a la asistencia sanitaria, ambos territorios ofrecen cifras de equipamiento muy parecidas, incluso el número de hospitales en ambas zonas en 2012 es exactamente el mismo: 37. La situación por dependencia funcional no ofrece diferencias demasiado dispares ni tampoco el número de camas. En cuanto a quirófanos en funcionamiento, en 2012, Galicia registra 10 por cada 100.000 habitantes y Castilla y León, 9. La proporción de quirófanos en centros públicos respecto a los que se encuentran en privados es muy similar en ambas regiones. Galicia, con 198 públicos y 74 privados, tiene 2,68 veces más del primer tipo que del segundo. Castilla y León ofrece un coeficiente casi idéntico: al contar con 157 públicos y 60 privados le sale una tasa de multiplicación entre unos y otros del 2,62 (Instituto de Información Sanitaria, 2009, 2013a).

No obstante, la estructura comunicativa es muy dispar. Castilla y León centraliza la comunicación de todos los hospitales públicos en el departamento de comunicación de la consejería de Sanidad del gobierno regional, mientras que en Galicia, aparte de disponer de un servicio de este tipo en la administración regional, también hay

Revista de Comunicación de la SEECI. (Marzo, 2016). Año XX (n 39), 105-126 
gabinetes de este tipo en cada gran hospital. De esta manera, en la primera región hay siete departamentos de comunicación (sumando públicos y privados), mientras que en la segunda hay quince.

El marco teórico utilizado es el de las relaciones públicas, centrado en los modelos, los roles, la definición de objetivos concretos, la participación en planes estratégicos, la influencia personal, los públicos, las herramientas, las fases de un programa de relaciones públicas, etc.

La parte empírica gira en torno a tres herramientas diferentes. Por una parte, se han realizado entrevistas personales a los responsables de comunicación de los hospitales de las dos comunidades autónomas con el fin de obtener información cualitativa sobre su trayectoria, sus labores, su independencia, las mejoras que implantarían, etc. Por otro lado, esas mismas personas han cumplimentado una encuesta con la que se obtienen datos cuantitativos que ayudan a medir el uso de las relaciones públicas en el ámbito hospitalario. Asimismo, se ha realizado un análisis de prensa para conocer si existen diferencias en la exposición que tienen los temas sanitarios en los medios de comunicación según las características comunicativas de cada región. Finalmente, se han consultado varias encuestas sobre el interés que tiene la población sobre estos asuntos y su satisfacción con el funcionamiento del sistema sanitario.

De esta manera, la parte empírica de la tesis doctoral conjuga tanto el paradigma cualitativo como el cuantitativo. El primer enfoque persigue crear tendencias generalizables y sus datos son susceptibles de tratamiento estadístico (Lozano Rendón, 2007). Por el contrario, el segundo ayuda a contextualizar y encuadrar hipótesis, algo que no se puede realizar desde un punto de vista exclusivamente cuantitativo (Wimmer y Dominick, 1996, p. 148).

Con la unión de ambas metodologías se logra el fenómeno de la triangulación, un método que se está convirtiendo en un esquema común de las empresas y las universidades (Casetti y Di Chio, 1999, p. 333), ya que compensa las debilidades de un método con otro distinto y así se consiguen los resultados "más fiables y enteramente satisfactorios" (Elías, 2000, p. 38). Se trata de la fase más avanzada de la integración de varias metodologías (complementación, combinación y triangulación), puesto que convergen los resultados de todas las metodologías para que alcancen las mismas conclusiones (Rodríguez Breijo, 2009). Se produce una triangulación metodológica cuando se emplean al menos dos técnicas de investigación (pueden ser cualitativa y cualitativa) o dos metodologías distintas con el objetivo de conocer y analizar un mismo objeto. A continuación se analiza detalladamente cada análisis utilizado en la investigación.

\subsection{Análisis cualitativo: entrevistas a los responsables de comunicación de los hospitales}

Revista de Comunicación de la SEECI. (Marzo, 2016). Año XX (n 39), 105-126 
Con el ánimo de conocer las características propias de la comunicación en los hospitales de Castilla y León y de Galicia, se han realizado entrevistas personales en profundidad a los responsables de comunicación de estos centros, tanto públicos como privados. Para ello, se consultó el Catálogo Nacional de Hospitales (Ministerio de Sanidad, 2013) con el fin de conocer los centros de atención especializada de cada zona.

A los responsables de comunicación de los hospitales públicos se los localizó a través de los departamentos de comunicación de la consejería de Sanidad de cada comunidad. Por su parte, a los encargados de comunicación de los centros privados se les encontró a través de las secciones de información de cada hospital, que derivaron la consulta a los empleados en plantilla o a las empresas externas, según el caso. Anteriormente, se creó una página web con información sobre la tesis doctoral para que los responsables de comunicación conociesen con más detalle las características del estudio. En esta plataforma se incluyó un resumen general, los objetivos, la estructura, los currículum vítae abreviados de los directores y de la autora y los datos de contacto. A la hora de solicitar la participación de los responsables de comunicación, se les explicó por teléfono las particularidades de la tesis doctoral y se les envió por correo electrónico un resumen del trabajo y un enlace a la página web.

Sumando los públicos y los privados, para los 37 hospitales que tiene cada región, Castilla y León cuenta con 7 departamentos de comunicación (uno público y seis privados) y Galicia, con 15 (8 públicos y 7 privados). De estos 22 responsables de comunicación, 19 han aceptado colaborar. Divididos por comunidad y titularidad, en Castilla y León han participado 3 privados y uno público, mientras Galicia han sido 8 de hospitales públicos y 7 de privados. Los encuentros en Galicia se produjeron durante los meses de mayo y junio de 2013, mientras que en Castilla y León se produjeron en marzo de 2014. Como los pertenecientes al Sistema Nacional de Salud en Castilla y León no cuentan con una persona dedicada a la comunicación, sino que centralizan toda esta labor en el departamento de comunicación de la Consejería de Sanidad, se entrevistó a la jefa de este servicio.

\subsubsection{Contenido de las entrevistas}

En los encuentros se trataron temas como los estudios que habían cursado, la trayectoria profesional, su día a día, los principales públicos y las acciones con cada uno de ellos, la libertad o presiones que sufren, etc. Los encuentros tenían una duración de entre 45 minutos a una hora y media, según la disponibilidad de los entrevistados y el desarrollo de las entrevistas. Las preguntas generales sobre las que giraba la charla, y a partir de las cuales podían surgir otras nuevas, son las siguientes:

1. Trayectoria profesional.

2. Día a día en el hospital.

3. Principales públicos, a saber: pacientes, familiares y visitas, empleados, agentes políticos e institucionales, inversores o accionistas (en su caso), medios de comunicación, población en general... 
4. ¿Cómo se comunica con cada público?

5. ¿Alguna acción de comunicación novedosa?

6. Organigrama: ¿de quién depende el departamento de comunicación?

7. ¿Influye la persona que gobierne la comunidad o el gerente del hospital? Ejemplos.

8. ¿Conoce su presupuesto? ¿Le supone una gran traba?

9. ¿Cuánta libertad tiene para realizar las acciones (contenidos, presupuesto, ideología...)?

10. ¿Cuáles son las presiones que tiene que soportar (derecho a la intimidad, derecho a la información...)?

11. ¿Qué diferencias cree que existen entre gestionar la comunicación dentro de la institución o a través de una empresa externa? ¿Qué considera que es mejor?

12. Beneficios de un departamento de comunicación para el hospital y la sanidad en general.

13. Errores comunicativos que se comenten (tanto en el hospital como en el ámbito de la salud en general).

14. Mejoras que implantaría (tanto en el hospital como en general).

15. ¿Por qué no se llevan a cabo esas acciones (tanto en el hospital como en general)?

Para el análisis de esta parte, se agrupan sus opiniones según la comunidad autónoma y la temática y se han incorporado declaraciones textuales así como ejemplos reales que han proporcionado los trabajadores. En algunos casos, bien por petición expresa de los encuestados o por iniciativa propia de la investigadora, no se atribuyen las citas a un personaje en concreto por las repercusiones que podrían tener en su entorno.

\subsection{Análisis cuantitativo: encuestas a los responsables de comunicación de los hospitales}

Dado que las entrevistas personales proporcionan valoraciones únicamente cualitativas, se ha diseñado un cuestionario con preguntas referentes a las relaciones públicas y se les ha entregado a los responsables de comunicación de los hospitales para que lo cumplimentasen. De esta manera, se obtienen datos cuantitativos y analizables estadísticamente acerca de los siguientes aspectos: los modelos, los roles, el modelo de influencia personal, el plan de comunicación, la participación en planes estratégicos, los públicos objetivo, las fases de un programa de relaciones públicas, los tipos de evaluación y la preparación ante crisis.

En la mayoría de los casos, lo rellenaron el mismo día de la entrevista y, en algunos pocos casos y debido al poco tiempo disponible para atender a la investigadora, lo cumplimentaron más adelante a través de un servidor online. Hubo una persona que, alegando falta de tiempo, no respondió al cuestionario ni de forma presencial ni online. 
Algunas preguntas han sido redactadas por la investigadora de esta tesis y otras han sido adaptadas de otras investigaciones relacionadas. Se ha optado por cuatro clases de recogida de datos. En su mayoría, se trata de preguntas de escala Likert de 1 a 5, donde el uno equivale a "totalmente en desacuerdo" y el cinco a "totalmente de acuerdo". También hay varias tablas de verificación o checkboxs, para las cuales el entrevistado únicamente debe seleccionar las variables con las que esté de acuerdo dentro de unas opciones fijas. Asimismo, unas preguntas se responden 0 con respuesta "sí o no" y en unos pocos casos, se debe seleccionar la respuesta de una lista desplegable.

Las preguntas para determinar el uso de los cuatro modelos de J. E. Grunig (1984) han sido adaptadas del cuestionario del Excellence Study, empleado también en otras investigaciones, como las de Gordon y Kelly (1999) y Xifra (2008). Así, se ofrecen cuatro ideas por cada modelo estudiado, dando un total de 16 preguntas. También se ha querido estudiar la presencia del bidireccional simétrico persuasivo, propuesto por José Luis Arceo Vacas (2006), por lo que se han redactado otras cuatro afirmaciones para determinar su uso, quedando la cifra, finalmente, en 20, pudiendo ser contestados en una escala de 1 a 5.

En cuanto a los roles de relaciones públicas empleados, se ha tenido en cuenta la selección que inicialmente realizó Kelly (1994) y que posteriormente se adaptaría en otras investigaciones, como la de Gordon y Kelly (1999). Esta investigadora redujo las 16 preguntas de J. E. Grunig (1992) a ocho, cuatro por cada rol estudiado. En este caso, también se ha empleado una escala Likert.

La siguiente serie de afirmaciones de la encuesta trata sobre la definición y consecución de objetivos concretos y sobre la participación en planes estratégicos. Las referentes a la primera cuestión son de elaboración propia, mientras que las del segundo aspecto se han tomado de Gordon y Kelly (1999). Más concretamente, las dos ideas que se han seleccionado de estas investigadoras son: el equipo estratégico de planes suele consultar al departamento de comunicación y algún miembro del departamento forma parte del equipo estratégico del hospital. Exceptuando una respuesta de "sí o no", el resto se han diseñado mediante escala.

Para determinar el modelo de influencia personal propuesto por Sriramesh (1991), se ha empleado el cuestionario utilizado por Xifra (2008) en su análisis de los modelos de relaciones públicas en Cataluña, que incluye las siguientes cuestiones: tengo buenas relaciones interpersonales con otros empleados, tengo buenas relaciones interpersonales con las personas externas de mi organización, las relaciones sociales es una de mis actividades más importantes y procuro beneficios (comidas, regalos) para ganar influencia con mis contactos personales. Los encuestados podían responder en una escala de 1 ("totalmente en desacuerdo") a 5 ("totalmente de acuerdo").

Una vez determinada la importancia que los responsables de comunicación le otorgan a las relaciones sociales, se ha querido comprobar qué público es el más relevante

Revista de Comunicación de la SEECI. (Marzo, 2016). Año XX (n 39), 105-126 
para el departamento. Para ello, se ha seleccionado una serie de colectivos que, a priori, engloba todas las audiencias de los hospitales. Estos son: los pacientes, los familiares y las visitas, los empleados, los agentes políticos e institucionales, los inversores o accionistas (en su caso), los medios de comunicación y la población en general. De esta lista, los encuestados debían elegir de 1 a 5 el nivel de importancia que otorgan a cada colectivo.

Dado que una gestión estratégica de las relaciones públicas exige una planificación concreta, se ha querido determinar el grado de relevancia que se le otorgan a las diferentes fases mediante una escala. De los modelos existentes, se ha seleccionado la versión de Hainsworth y Wilson (1992) y Guth y Marsh (2000), que dividen las etapas de una campaña en: investigación, planificación, comunicación y evaluación.

Para hallar las herramientas que usan los departamentos de comunicación en cada etapa, se han creado unas tablas de verificación o checkboxs con una amplia lista de opciones. Asimismo, se ofrecía la posibilidad de completar estas respuestas con una casilla de libre cumplimentación. En cuanto a la fase de investigación, muchas de las tácticas se han seleccionado del manual Relaciones Públicas: estrategias y tácticas, de Wilcox, Cameron y Xifra (2007), mientras que otras son de elaboración propia. En cuanto a la planificación, todas ellas provienen del citado manual. Dada la cantidad de herramientas disponibles para ejecutar la fase de comunicación y los públicos a los que se puede dirigir, se ha dividido las tablas de verificación según las audiencias principales para las que se utilizan: empleados, pacientes y visitas, medios de comunicación y población. Así, tanto para esta sección como para la de evaluación, se han consultado y adoptado algunas opciones de Wilcox, Cameron y Xifra (2007), Xifra (2007), Castillo Esparcia (2006), Rojas (2008), Palencia-Lefler (2011) y otras de inclusión propia.

Asimismo, se han estudiado, tal y como Wilcox, Cameron y Xifra (2007) dividen, los diferentes tipos de medición: producción, exposición, opinión y actitud del público y acciones y comportamientos del público. Para ello, se ha utilizado una escala Likert. Posteriormente, se ha demandado a los responsables de comunicación si poseen un plan de crisis, cada cuánto se revisa y se pone en práctica (estas dos preguntas con respuestas fijas de una lista desplegable) y si existe un equipo prefijado de personas para posibles conflictos. Finalmente, se les ha preguntado cómo de importante consideran su departamento para el hospital y cómo de satisfechos están con las actividades de comunicación que lleva a cabo el centro en una escala de 1 a 5.

Una vez las encuestas han sido cumplimentadas, se han introducido las respuestas en el programa estadístico SPSS y se han realizado diversos análisis, sobre todo descriptivos y frecuencias, dividiendo los resultados según las hipótesis de partida. También se han ejecutado varias pruebas estadísticas, como la prueba de chicuadrado o la prueba $\mathrm{T}$ de Student, para hallar las relaciones estadísticamente significativas. La significación se ha fijado en igual o menor de 0,05. De esta forma, se 
puede establecer que existe una relación estadística y que los resultados no son frutos del azar. Así, se ha analizado la diferencia de las respuestas entre Castilla y León y Galicia, entre el ámbito público y el privado y entre los gabinetes internos y externos.

\subsection{Análisis de prensa: medición de la exposición}

Para estudiar la repercusión que tienen los departamentos de comunicación en el número y calidad de las informaciones sobre salud y sanidad que publican los medios de comunicación, se ha procedido a analizar las noticias que publican los periódicos de cada comunidad. Para ello, se ha empleado la herramienta Iconoce, una base de datos de medios de comunicación con un servicio de hemeroteca que permite hacer búsquedas introspectivas de las noticias en internet desde el año 2001 (Iconoce, 2014). El periodo de estudio se ha limitado al año 2012 y se han seleccionado todos los periódicos generalistas regionales, provinciales y locales de ambas comunidades que mantienen el acceso abierto a ese espacio. De esta manera, los lugares web consultados en cada comunidad ascienden a 15, dando un total de 30 periódicos analizados.

Dado que muy pocos diarios electrónicos poseen una sección fija dedicada a la salud, las noticias que componen la muestra del estudio han sido obtenidas buscando a través de varias palabras clave. En el caso de Castilla y León, los vocablos elegidos han sido: sanidad, salud, medicina, médico, hospital, ambulatorio, farmacia, farmacéutico, enfermedad y Sacyl. Para encontrar las noticias de Galicia, se ha añadido la traducción al gallego de los términos en español en los casos en los que difiere, así como el Servicio Galego de Saúde (Sergas), de tal manera que se han buscado las informaciones a través de la siguiente cadena: sanidad, salud, sanidade, saúde, medicina, médico, hospital, ambulatorio, farmacia, farmacéutico, enfermedad, enfermidade y Sergas.

\subsubsection{Muestreo y margen de error}

Puesto que Iconoce ofrece únicamente 200 resultados como máximo en una sola búsqueda $y$, en consecuencia, es muy complicado determinar el número de noticias que publican los medios de comunicación sobre salud en un periodo largo de tiempo, se ha procedido a realizar un muestreo para calcular las noticias que divulgan los diferentes medios en un año, más concretamente en 2012. Para ello, se ha seleccionado al azar un día de cada uno de los doce meses del año, intentando, además, que se tratara de diferentes semanas y días de la semana. Se ha empezado con un martes de la primera semana de enero, se ha continuado con un miércoles de la segunda semana de febrero, se ha proseguido con un jueves de la tercera semana de marzo y así sucesivamente.

El número total de noticias recopiladas en los 12 días que componen el muestreo asciende a 713 en Castilla y León y 842 en Galicia, o lo que es lo mismo: 59 noticias diarias de media en la primera región y 70 en la segunda. 
De esta forma, el muestreo determina que en Castilla y León hay 21.687 noticias sobre salud en un año y en Galicia, 25.611. Para conseguir una muestra que sea representativa según el universo de estudio, se ha examinado el tamaño de la muestra según el tamaño del universo y el margen de error deseado.

\begin{tabular}{|l|l|l|l|l|l|l|}
\hline \multirow{2}{*}{$\begin{array}{l}\text { Tamaño } \\
\text { del } \\
\text { universo }\end{array}$} & \multicolumn{6}{|c|}{ Tamaño de la muestra según el margen de error aceptado } \\
\cline { 2 - 7 } & $\mathbf{1 \%}$ & $\mathbf{2 \%}$ & $\mathbf{3 \%}$ & $\mathbf{4 \%}$ & $\mathbf{5 \%}$ & $\mathbf{1 0 \%}$ \\
\hline 500 & - & - & - & - & 222 & 83 \\
\hline 1.000 & - & - & - & 385 & 286 & 91 \\
\hline 1.500 & - & - & 638 & 441 & 316 & 94 \\
\hline 2.000 & - & - & 714 & 476 & 333 & 95 \\
\hline 2.500 & - & 1.250 & 769 & 500 & 345 & 96 \\
\hline 3.000 & - & 1.364 & 811 & 517 & 353 & 97 \\
\hline 3.500 & - & 1.458 & 843 & 530 & 359 & 97 \\
\hline 4.000 & - & 1.538 & 870 & 541 & 364 & 98 \\
\hline 4.500 & - & 1.607 & 891 & 549 & 367 & 98 \\
\hline 5.000 & - & 1.667 & 909 & 556 & 370 & 98 \\
\hline 6.000 & - & 1.755 & 938 & 566 & 375 & 98 \\
\hline 7.000 & - & 1.842 & 949 & 574 & 378 & 99 \\
\hline 8.000 & - & 1.905 & 976 & 580 & 381 & 99 \\
\hline 9.000 & - & 1.957 & 989 & 584 & 383 & 99 \\
\hline 10.000 & 5.000 & 2.000 & 1.000 & 588 & 385 & 99 \\
\hline 15.000 & 6.000 & 2.143 & 1.034 & 600 & 390 & 99 \\
\hline 20.000 & 6.667 & 2.222 & 1.053 & 606 & 392 & 100 \\
\hline 25.000 & 7.143 & 2.273 & 1.064 & 610 & 394 & 100 \\
\hline 50.000 & 8.333 & 2.381 & 1.087 & 617 & 397 & 100 \\
\hline 100.000 & 9.091 & 2.439 & 1.099 & 621 & 398 & 100 \\
\hline$\infty$ & 10.000 & 2.500 & 1.111 & 625 & 400 & 100 \\
\hline
\end{tabular}

Tabla 1. Tamaño de la muestra según distintos márgenes de error y el tamaño del universo. Fuente: Arkin y Colton, Tables for Statisticians. Tomado de J. Bugeda, Manual de Técnicas de Investigación Social. I.E.P. Madrid, 1970.

Para obtener un margen de error del $5 \%$ y que la muestra sea significativa, se han de examinar entre 392 y 394 noticias de Castilla y León y entre 394 y 397 de Galicia. Con el fin de redondear, se ha acordado estudiar 400 de cada territorio. A grandes rasgos, los elementos que se han estudiado del análisis de prensa son los siguientes: el número de noticias, la sección, los autores, el lugar, el género, el tamaño, los audiovisuales, el tema, la neutralidad, las organizaciones informadoras, el contraste, el archivo, las segundas fuentes, los enlaces e la interacción del lector.

Los resultados se han introducido en el programa estadístico SPSS y se han realizado diversos análisis, como frecuencias, descriptivos, tablas de contingencia, prueba de chi-cuadrado, prueba T de Student, etc. La significación en las pruebas estadísticas se 
ha fijado en igual o menor de 0,05 . De esta forma, se puede establecer que existe una relación estadística y que los resultados no son frutos del azar.

\subsection{Medición de la opinión y actitud del público}

Con el fin de conocer la opinión y la actitud de la población hacia la sanidad, se ha hecho uso del Barómetro Sanitario que publica anualmente el Ministerio de Sanidad, Servicios Sociales e Igualdad, y cuyos últimos resultados según la fecha de la investigación corresponden al ejercicio 2013. Se trata de un informe que recoge la valoración de los ciudadanos sobre el funcionamiento del sistema sanitario español y su satisfacción con la calidad de las prestaciones, en los dos casos en referencia al ámbito público. Es el único que divide los resultados por comunidades autónomas y permite la comparación entre las dos regiones del estudio. La muestra total se compone de 7.800 entrevistas con cuestionario estructurado realizadas de manera personal en los domicilios de 237 municipios de las 17 comunidades y de las dos ciudades autónomas (Instituto de Información Sanitaria, 2013b). Posee un error de muestreo, en conjunto, del $\pm 1,13 \%$ para un nivel de confianza del $95,5 \%$. En Castilla y León se realizaron 423 entrevistas para el último número y en Galicia, 441 (Instituto de Información Sanitaria, 2013c).

De este informe se han analizado dos aspectos en los que las relaciones públicas pueden tener implicación: la opinión y actitud, y las acciones y comportamientos del público. Para hallar el primer aspecto, se ha consultado el interés que tiene la sanidad tanto para los castellano-leoneses como para los gallegos, así como la valoración que realizan del sistema sanitario y su satisfacción con diferentes aspectos. Por el contrario, para determinar las acciones y comportamientos de los ciudadanos, se han utilizado las mediciones acerca de la elección de los hospitales y de los motivos que impulsan a tener esa determinación. Este último análisis divide las elecciones por centros de dependencia pública y privada. Así, se contraponen los resultados de Castilla y León y de Galicia para comprobar si hay diferencias considerables.

\section{DISCUSIÓN}

La utilización de varios métodos de análisis permite obtener unos resultados más fiables de lo que hubiera ofrecido una sola metodología. El análisis de las entrevistas personales y de las encuestas no muestra discrepancias reseñables y el análisis de ambas metodologías proporciona unos resultados similares. Así, se ha podido comprobar que el uso de las relaciones públicas en muy similar en las dos comunidades estudiadas. En ambos casos, el empleo que hacen de los roles y modelos es prácticamente el mismo. Los comunicadores de los dos territorios también tienen la misma posibilidad de formar parte del equipo estratégico y de disponer de un plan y de un equipo prefijado para afrontar posibles crisis. Los tres principales públicos son los mismos en los dos territorios, aunque cambien ligeramente de orden. En las dos regiones se presta más atención a la comunicación externa, sobre todo la canalizada a través de los medios de comunicación, dejando como asignatura pendiente la interna. Asimismo, utilizan similares herramientas en las distintas etapas de un programa de relaciones públicas, aunque los gallegos emplean un número

Revista de Comunicación de la SEECI. (Marzo, 2016). Año XX (n 39), 105-126 
mayor en la fase de comunicación. La única diferencia estadísticamente significativa se ha detectado en Castilla y León, cuyos comunicadores otorgan una mayor importancia a las relaciones personales.

Tras las entrevistas personales y las encuestas a los responsables de comunicación de los hospitales se observa que la mayor diferencia en el uso de las relaciones públicas se encuentra según la titularidad del centro. Así, los públicos suelen prestar más atención a los medios de comunicación y a los agentes políticos e institucionales que los privados. Estos segundos, en cambio, se centran más en las relaciones personales, son más propensos a formar parte de la coalición estratégica y a disponer de un plan y equipo prefijado para posibles crisis. También son más conscientes de las diferentes etapas de un programa de relaciones públicas y evalúan sus acciones más frecuentemente que los trabajadores del ámbito público. Asimismo, presentan un mayor abanico de actividades novedosas, tanto en lo referente a nuevas tecnologías como en la organización de eventos.

Las empresas externas tienden a formar parte del equipo estratégico de planes más a menudo que las personas que trabajan internamente. Asimismo, suelen guiarse por un plan de comunicación y prestan más atención a las distintas fases de un programa de relaciones públicas, con una mayor diferencia en la planificación y evaluación. Así, suelen guiarse por objetivos y echan mano de más herramientas y de diferentes enfoques teóricos para analizar el éxito de sus esfuerzos comunicativos.

El número de noticias que publican los periódicos de cada comunidad relativas a la salud y sanidad es prácticamente idéntica. El uso de audiovisuales y de enlaces, así como de datos de archivo, contraste y segundas fuentes tampoco muestran diferencias reseñables. En ambos casos, hay un alto número de fuentes y de lugares de origen de la noticia. No obstante, en Galicia, donde hay más departamentos de comunicación sobre estos asuntos, las noticias suelen insertarse con más frecuencia en la sección local, las novedades se enmarcan más a menudo en lugares pertenecientes a la región y es más probable que los textos sean elaborados por los redactores del periódico en vez de por agencias de comunicación. Además, suelen divulgarse más composiciones explicativas e interpretativas, más allá de la mera información. En cuanto a los hospitales, son fuentes de información más recurrentes en Galicia que en Castilla y León, tanto en los géneros informativos como en los no informativos.

Aunque los lectores castellano-leoneses comentan con un poco más de frecuencia que los gallegos las noticias sobre salud y sanidad aparecidas en los periódicos de internet, estos últimos adelantan a los primeros en frecuencia y número de votos. También recomiendan las noticias con una mayor asiduidad en la red social Facebook y comparten más a menudo y con un número mayor las noticias en Twitter. La primera y las dos últimas relaciones resultan ser, de hecho, estadísticamente significativas. 
Los ciudadanos gallegos consideran la sanidad como un tema de mayor trascendencia que los castellano-leoneses. Asimismo, realizan una mejor valoración del sistema público, una visión que, además, mejora con el paso de los años. Por ello, más gallegos elegirían los centros públicos antes que los castellano-leoneses y exponen más motivos para justificarlo. Sin embargo, cuando se trata de satisfacción relativa a la atención médica especializada, la percepción de los ciudadanos que acudieron a un médico especialista es más positiva en Castilla y León, a pesar de que para aquellos que acudieron a un hospital es más alta en Galicia. En cuanto a la satisfacción general con el sistema sanitario público, los castellano-leoneses adelantan a los gallegos.

Dados los resultados conseguidos, se pueden dar respuesta a las hipótesis de partida. De las seis propuestas, se han aceptado dos, se ha refutado una y las tres restantes se han aceptado parcialmente:

H1. Debido a la competencia comunicativa, cuando una gran parte de los hospitales de una región posee departamentos de comunicación, las relaciones públicas que se adoptan deberían ser más excelentes: refutada.

H2. La presencia de departamentos de comunicación en los hospitales debe tener una repercusión en el número y calidad de las informaciones sobre salud y sanidad que publican los medios de comunicación de la región: parcialmente aceptada.

H3. Los ciudadanos de las zonas que cuentan con departamentos de comunicación en la mayor parte de los hospitales han de ser más activos en el consumo de información sanitaria y salud, en el sentido de que comentan más frecuentemente estos temas, los valoran y los comparten con sus conocidos: parcialmente aceptada:

H4. Los ciudadanos de las zonas en las que existen más departamentos de comunicación deberían tener una mejor valoración y un grado de satisfacción más alto hacia el sistema sanitario: parcialmente aceptada.

H5. Los departamentos de comunicación de los hospitales públicos y de los privados pueden tener diferencias en cuanto a su interés por la opinión pública, en el uso de métodos más o menos tradicionales y en las nuevas acciones de comunicación: aceptada.

H6. Los hospitales gestionados por empresas externas de comunicación ejercerían unas relaciones públicas más excelentes que aquellos que cuentan con uno o dos empleados en plantilla debido a la diversidad de personal: aceptada.

\section{CONCLUSIONES}

La utilización de la triangulación en el análisis de la comunicación que se realiza desde varias entidades, en este caso sanitarias, obtiene beneficiosos resultados, puesto que

Revista de Comunicación de la SEECI. (Marzo, 2016). Año XX (n 39), 105-126 
permite contrarrestar las debilidades de cada método, ofreciendo unos resultados más fiables. En este caso, se han comparado los datos cualitativos recogidos durante la entrevista personal con la información cuantitativa derivada de la encuesta, con el análisis de prensa y con el análisis de la opinión y comportamientos del público. Gracias a ello se han podido redactar varias conclusiones que resumen las contribuciones de esta tesis doctoral al ámbito de las relaciones públicas y de la comunicación sanitaria:

1. No hay evidencias de que en un territorio se adopten relaciones públicas excelentes en el ámbito de la salud como consecuencia de una gran competencia comunicativa.

2. La mayor diferencia en el uso de las relaciones públicas se ha detectado según la titularidad del hospital. Los responsables de comunicación de los centros privados tienden a hacer un mejor uso de esta disciplina que los de los públicos.

3. En general, las empresas de comunicación externas que se encargan de la comunicación de un hospital hacen un uso más excelente de las relaciones públicas que las personas que trabajan como un empleado interno del centro.

4. El número de noticias que publican los medios de comunicación sobre salud y sanidad no tiene relación con la cantidad de departamentos de comunicación que existen en un determinado territorio. Es decir, el interés por la sanidad por parte de los periodistas no depende del número de departamentos de comunicación, puesto que pueden conseguir datos de otras fuentes.

5. Aunque el número de noticias en los periódicos es similar independientemente del cómputo de departamentos de comunicación de una zona, la calidad de las informaciones es superior en los lugares que cuentan con un mayor número de gabinetes de comunicación.

6. En aquellas zonas con una mayor suma de departamentos de comunicación dedicados a la sanidad los ciudadanos votan y comparten más a menudo las informaciones de internet relativas a la salud, aunque no las comenten más frecuentemente.

7. Los ciudadanos de las zonas con un mayor número de departamentos de comunicación en el terreno sanitario consideran este tema más trascendente que aquellos que cuentan con menos gabinetes de este tipo.

8. En las regiones con un mayor cómputo de hospitales públicos con departamentos de comunicación, los ciudadanos tienen una mayor valoración de la sanidad pública y elegirían antes esta titularidad y debido a una mayor cantidad de motivos. 
9. En cuanto a satisfacción con el sistema sanitario público, no existen diferencias entre los ciudadanos independientemente del número de departamentos de comunicación que hay en un territorio.

\section{REFERENCIAS}

Amador Romero, F. J. (2004). Medios de comunicación y opinión pública sanitaria. Atención Primaria, 33(2), 95-98. doi: http://dx.doi.org/10.1157/13057251

Anand, R. C., \& Chakravarti, A. (1981). Public Relations in Hospital. Health and Population (Perspectives \& Issues), 4(4), 252-259.

Arceo Vacas, J. L. (2006). La investigación de relaciones públicas en España. Anàlisi: Quaderns de comunicació i cultura, (34), 111-124.

Avery, E. J., Lariscy, R. W., Kim, S., \& Hocke, T. (2010). A quantitative review of crisis communication research in public relations from 1991 to 2009. Public Relations Review, 36(2), 190-192. doi: http://dx.doi.org/10.1016/j.pubrev.2010.01.001

Avery, E., Lariscy, R., Amador, E., Ickowitz, T., Primm, C., \& Taylor, A. (2010). Diffusion of Social Media Among Public Relations Practitioners in Health Departments Across Various Community Population Sizes. Journal of Public Relations Research, 22(3), 336-358.

Balima, S. T. (2006). Comunicación para la salud en África: experiencias y desafíos. Comunicar: Revista científica iberoamericana de comunicación y educación, (26), 21-26.

Brodie, R. J., Glynn, M. S., \& Van Durme, J. (2002). Towards a Theory of Marketplace Equity: Integrating Branding and Relationship Thinking with Financial Thinking. Marketing Theory, 2(1), 5-28.

Cangelosi, J. D., Ranelli, E., \& Markham, F. S. (2009). Who is Making Lifestyle Changes Due to Preventive Health Care Information? A Demographic Analysis. Health Marketing Quarterly, 26(2), 69-86. doi: http://dx.doi.org/10.1080/07359680802619776

Casetti, F., \& Di Chio, F. (1999). Análisis de la televisión: instrumentos, métodos y prácticas de investigación. Barcelona: Paidós.

Castiel, L. D., \& Álvarez-Dardet, C. (2005). Las tecnologías de la información y la comunicación en salud pública: las precariedades del exceso. Revista española de salud pública, 79(3), 331-337.

Castillo Esparcia, A. (2006). Las relaciones públicas internas como factor de gestión empresarial. Anàlisi: Quaderns de comunicació i cultura, (34), 193-208. 
Centro de Investigaciones Sociológicas. (2012). Barómetro sanitario 2012 (total oleadas). Recuperado el 15 de septiembre de 2015 de http://www.cis.es/cis/opencm/ES/1_encuestas/estudios/ver.jsp?estudio=14096

Costa Sánchez, C. (2012). El gabinete de comunicación del hospital. Propuesta teórica y acercamiento a la realidad de los departamentos de comunicación de los hospitales públicos de Galicia. Doxa Comunicación: revista interdisciplinar de estudios de comunicación y ciencias sociales. Universidad San Pablo-CEU.

Del Pozo Irribarría, J., \& Ferreras Oleffe, M. (2011). La telefonía móvil como instrumento de comunicación para la información y prevención del consumo de drogas. In U. Cuesta Cambra, T. Menéndez Hevia, \& A. Ugarte Iturrizaga (Eds.), Comunicación y salud: nuevos escenarios y tendencias (pp. 111-123). Madrid: Editorial Complutense.

Díaz, H. A. (2011). La comunicación para la salud desde una perspectiva relacional. In U. Cuesta Cambra, T. Menéndez Hevia, \& A. Ugarte Iturrizaga (Eds.), Comunicación y salud: nuevos escenarios y tendencias (pp. 33-49). Madrid: Editorial Complutense.

Eggener, S. (1998). The power of the pen: Medical journalism and public awareness. Journal of the American Medical Association, 279(17), 1.400.

Elías, C. (2000). Flujos de información entre científicos y prensa. Universidad de La Laguna.

Freberg, K., Palenchar, M. J., \& Veil, S. R. (2013). Managing and sharing H1N1 crisis information using social media bookmarking services. Public Relations Review, 39(3), 178-184.

Gbadeyan, R. A. (2010). Health Care Marketing and Public Relations in Not for Profit Hospitals in Nigeria. International Journal of Business and Management, 5(7), 117-125.

Gordon, C. G., \& Kelly, K. S. (1999). Public Relations Expertise and Organizational Effectiveness: A Study of U.S. Hospitals. Journal of Public Relations Research, 11(2), 143-165. doi: http://dx.doi.org/10.1207/s1532754xjprr1102_03

Grunig, J. E. (1984). Organizations, environments, and models of public relations. Public Relations Research \& Education, 1(1), 6-29.

Grunig, J. E. (1992). Excellence in Public Relations and Communication Management (2nd ed.). Hillsdale (New Jersey): Lawrence Erlbaum Associates. 
Gudykunst, W. B., \& Kim, Y. Y. (1992). Communicating with strangers: An approach to intercultural communication. New York: McGraw-Hill.

Guth, D. W., \& Marsh, C. (2000). Public Relations: A Values-Driven Approach. Boston: Allyn and Bacon.

Guy, B., Williams, D. R., Aldridge, A., \& Roggenkamp, S. D. (2007). Approaches to organizing public relations functions in healthcare. Health Marketing Quarterly, 24(3-4), 1-18. doi: http://dx.doi.org/10.1080/07359680802118969

Hainsworth, B. E., \& Wilson, L. J. (1992). Strategic program planning. Public Relations Review, 18(1), 9-15.

Henderson, J. K. (2005). Evaluating public relations effectiveness in a health care setting. Journal of Health and Human Services Administration, 28(1-2), 282-321.

Hibbard, J. H., Stockard, J., \& Tusler, M. (2005). Hospital performance reports: Impact on quality, market share, and reputation. Health Affairs, 24(4), 1.150-1.160.

Iconoce. (2014). Conócenos. Recuperado el 21 de octubre de 2014 de http://info.iconoce.com/servicios.php

Igartua Perosanz, J. J. (2006). Comunicación para la salud y sida: la aproximación educación-entretenimiento. Comunicar. Revista Científica de Comunicación y Educación, 26, 35-42.

Instituto de Información Sanitaria. (2009). Estadística de establecimientos sanitarios con régimen de internado. Indicadores hospitalarios. Evolución 2000-2008. Recuperado el 1 de septiembre de 2015 de https://www.msssi.gob.es/estadEstudios/estadisticas/docs/Evolutivo_20002008.pdf

Instituto de Información Sanitaria. (2013a). Estadística de centros sanitarios de atención especializada. Recuperado el 1 de septiembre de 2015 de http://www.msssi.gob.es/estadEstudios/estadisticas/estHospiInternado/inforAnual /homeESCRI.htm

Instituto de Información Sanitaria. (2013b). Notas metodológicas del Barómetro Sanitario. Recuperado el 3 de septiembre de 2015 de https://www.msssi.gob.es/estadEstudios/estadisticas/docs/BS_2013/BS_2013Met odologia.pdf

Instituto de Información Sanitaria. (2013c). Ficha técnica del Barómetro Sanitario. Recuperado el 1 de septiembre de 2015 de https://www.msssi.gob.es/estadEstudios/estadisticas/docs/BS_2013/BS_2013Fich aTecnica3oleadas.pdf 
Instituto Nacional de Estadística (INE). (2012). Cuentas Económicas. Contabilidad Regional de España. Base 2008. Recuperado el 25 de marzo de 2015 de http://www.ine.es/jaxi/menu.do?type=pcaxis\&path=/t35/p010\&file=inebase\&L=0

Instituto Nacional de Estadística (INE). (2013). Padrón municipal. Recuperado el 25 de marzo de 2015 de

http://www.ine.es/jaxi/menu.do?type=pcaxis\&path=/t20/e260\&file=inebase\&L=0

Ivanov, B., Sims, J. D., \& Parker, K. A. (2013). Leading the Way in New Product Introductions: Publicity's Message Sequencing Success With Corporate Credibility and Image as Moderators. Journal of Public Relations Research, 25(5), 442-466. doi: http://dx.doi.org/10.1080/1062726X.2013.795862

Jiménez Pernett, J., Olry de Labry Lima, A., García Gutiérrez, J. F., \& Salcedo Sánchez, M. . (2009). Estudio sobre salud en Internet para adolescentes y jóvenes en la ciudad de Granada. In J. del Pozo Irribarría, L. Pérez Gómez, \& M. Ferreras Oleffe (Eds.), Adicciones y nuevas tecnologías de la información y de la comunicación: perspectivas de su uso para la prevención y el tratamiento (pp. 95-102). Logroño: Gobierno de La Rioja: Consejería de Salud y Servicios Sociales.

Keller, A. C., Bergman, M. M., Heinzmann, C., Todorov, A., Weber, H., \& Heberer, M. (2014). The relationship between hospital patients' ratings of quality of care and communication. International Journal for Quality in Health Care, 26(1), 26-33. doi: http://dx.doi.org/10.1093/intqhc/mzt083

Kelly, K. S. (1994). Fund-raising encroachment and potential of the public relations department in the nonprofit sector. Journal of Public Relations Research, 6, 1-22.

Kirdar, Y. (2007). The Role of Public Relations for Image Creating in Health Services: A Sample Patient Satisfaction Survey. Health Marketing Quarterly, 24(3-4), 33-53. Retrieved from http://dx.doi.org/10.1080/07359680802119017

Kreps, G. L. (2008). Strategic use of communication to market cancer prevention and control to vulnerable populations. Health Marketing Quarterly, 25(1-2), 204-216. doi: http://dx.doi.org/10.1080/07359680802126327

Kreps, G. L., Bonaguro, E. W., \& Query, J. L. (1998). The history and development of the field of health communication. In B. K. Duffy \& L. D. Jackson (Eds.), Health communication research: A guide to developments and directions (pp. 1-15). Westport (Connecticut): Greenwood Press.

Kreps, G. L., \& Kunimoto, E. N. (1994). Effective communication in multicultural health care settings. Newbury Park (California): Sage Publications. 
Kurtz, H. P. (1969). Public Relations for Hospitals. Springfield (Illinois): Charles C. Thomas Publisher.

Lariscy, R. W., Avery, E. J., \& Sohn, Y. (2010). Health Journalists and Three Levels of Public Information: Issue and Agenda Disparities? Journal of Public Relations Research, 22(2), 113-135. http://dx.doi.org/10.1080/10627260802640708

Len-Ríos, M. E., Hinnant, A., \& Park, S.-A. (2009). Understanding how health journalists judge public relations sources: A rules theory approach. Public Relations Review, 35(1), 56-65. doi: http://dx.doi.org/10.1016/j.pubrev.2008.09.019

Lozano Rendón, J. C. (2007). Teoría e investigación de la comunicación de masas. México: Pearson Educación.

Lupiáñez Villanueva, F. (2009). Internet y salud: una aproximación empírica a los usos de Internet relacionados con la salud. In J. del Pozo Irribarría, L. Pérez Gómez, \& M. Ferreras Oleffe (Eds.), Adicciones y nuevas tecnologías de la información y de la comunicación: perspectivas de su uso para la prevención y el tratamiento (pp. 103-118). Logroño: Gobierno de La Rioja: Consejería de Salud y Servicios Sociales.

Meath, M. (2006). Taking time to care: best practices in long-term care communications. Corporate Communications: An International Journal, 11(4), 336-352. doi: http://dx.doi.org/10.1108/13563280610713833

Menéndez Prieto, M. D., \& Vadillo Olmo, F. J. (2010). El plan de comunicación hospitalario: Herramienta de gestión sanitaria (p. 115). San Vicente (Alicante): Editorial Club Universitario.

Ministerio de Sanidad, S. S. e I. (2013). Catálogo Nacional de Hospitales 2013. Recuperado el 28 de junio de 2015 de http://www.msssi.gob.es/ciudadanos/prestaciones/centrosServiciosSNS/hospitales /docs/CNH2013.pdf

Observatorio de la Comunicación Científica. (2008). Medicina y salud en la prensa diaria. Informe Quiral 10 años. Barcelona. Recuperado el 7 de septiembre de 2014 de http://www.upf.edu/pcstacademy/_docs/Quiral10.pdf

Palencia-Lefler, M. (2011). 90 Técnicas de comunicación y relaciones públicas (Vol. Segunda, p. 528). Barcelona: Profit Editorial.

Pew Research Center for The People and the Press. (2004). Online news audience larger, more diverse: News audiences increasingly politicized. Washington, DC.

Pham, H. H., Coughlan, J., \& O'Malley, A. S. (2006). The impact of quality reporting programs on hospital operations. Health Affairs, 25(5), 1412-1422. 
Rodríguez Breijo, V. (2009). Nuevos retos para el estudio de los efectos de los medios de comunicación. Madrid: Comunicación del XI Foro Universitario de Investigación en Comunicación.

Roessler, P. (2007). Public health and the media - A never-ending story. International Journal of Public Health, 52(5), 259-260. doi: http://dx.doi.org/10.1007/s00038007-0226-1

Roessler, Patrick. (2007). Public health and the media - a never-ending story, 52, 259-260. doi: http://dx.doi.org/10.1007/s00038-007-0226-

Rojas Orduña, O. I. (2008). Relaciones públicas: La eficacia de la influencia. Madrid: Escuela Superior de Gestión Comercial y Marketing, ESIC.

Scott, J., Vojir, C., Jones, K., \& Moore, L. (2005). Assessing nursing homes' capacity to create and sustain improvement. Journal of Nursing Care Quality, 20(1), 36-42.

Springston, J. K., \& Champion, V. L. (2004). Public relations and cultural aesthetics: designing health brochures. Public Relations Review, 30(4), 483-491. doi: http://dx.doi.org/10.1016/j.pubrev.2004.08.005

Springston, J. K., \& Weaver Lariscy, R. A. (2005). Public Relations Effectiveness in Public Health Institutions. Journal of health and human services administration, $28(1 / 2), 218-245$.

Sriramesh, K. (1991). The impact of societal culture on public relations: An ethnographic study of south Indian organizations. University of Maryland, College Park, Maryland.

Thomas, R. K. (2005). Marketing Health Services (p. 469). Health Administration Pr.

Viswanath, K. (2006). Public communications and its role in reducing and eliminating health disparities. In G. Thompson, F. Mitchell, \& M. Williams (Eds.), Examining the health disparities research plan of the national institutes of health: Unfinished business (pp. 215-253). Washington D.C.: National Academies Press.

Weaver-Lariscy, R., \& Springston, J. K. (2007). Health crises and media relations: Relationship management-by-fire. Health Marketing Quarterly, 24(3-4), 81. doi: http://dx.doi.org/10.1080/07359680802119066

Wilcox, D. L., Cameron, G. T., \& Xifra, J. (2007). Relaciones públicas: estrategias y tácticas (Vol. $8^{\mathrm{a}}$, últim, pp. XXXI, 783; 775-778). Madrid etc.: Pearson Educacion. 
Wimmer, R. D., \& Dominick, J. R. (1996). La investigación científica de los medios de comunicación: una introducción a sus métodos (Vol. $1^{\text {a }}$ español, pp. XIII, 492). Barcelona: Bosch.

Wrenn, B. (2002). Contribution to Hospital Performance. Journal of Hospital Marketing \&, 14(1), 3-13.

Xifra, J. (2007). Técnicas de las relaciones públicas. Editorial UOC.

Xifra, J. (2008). Las relaciones públicas. Revista Latina de comunicación social (pp. 392-399). Barcelona: Ediciones UOC. doi: http://dx.doi.org/10.4185/RLCS-632008-789-392-399

Youmans, S. L., \& Schillinger, D. (2003). Functional health literacy and medication use: the pharmacist's role. The Annals of pharmacotherapy, 37(11), 1726-9. doi: http://dx.doi.org/10.1345/aph.1D070 